\title{
Patient Self-Referral for Radiologic Screening Tests: Clinical and Ethical Concerns
}

\author{
Joshua J. Fenton, MD, and Richard A. Deyo, MD, MPH
}

Retail marketing of radiologic screening tests is increasingly common in the United States. Without a physician referral, patients can now directly purchase screening computed tomography (CT) or ultrasound scans. In this article, we consider the clinical and ethical ramifications of widespread screening of low-risk populations with 4 commonly marketed tests: whole-body CT, CT-based heart scans, heel ultrasound for osteoporosis, and carotid duplex sonography for carotid stenosis. All the tests are too inaccurate for screening in low-risk populations, and none has been proven to lead to early, beneficial intervention. Screening could be harmful if false-positive tests lead to extensive or invasive diagnostic evaluation. Finally, widespread testing could increase health care costs with little public health benefit. Patients should probably avoid radiologic screening tests until the tests have been appropriately evaluated in controlled studies and recommended by unbiased national panels, such as the United States Preventive Services Task Force. Primary care physicians and their professional societies should emphasize the uncertain benefits and potential hazards of indiscriminate imaging among healthy, asymptomatic consumers. (J Am Board Fam Pract 2003;16:494-501.)

Entrepreneurs have recently recognized a market for radiologic screening tests. For a price, many Americans-without a physician referral—can now conveniently purchase a computed tomography (CT) scan or an ultrasound examination from private, stand-alone radiology corporations. Currently, CT screening exams, including whole-body scans and coronary heart scans, are available at more than 100 centers nationwide, and the popularity of screening scans has begun to attract traditional medical centers into the market. One company is reaching rural markets by trucking scanners from town to town, and another provides ultrasound exams in mobile vans in 43 states, including heel ultrasound for osteoporosis and carotid duplex sonography. With the growth of this cottage industry, family physicians may encounter patients presenting for help interpreting their results. Other

Submitted, revised, 28 February 2003.

From the Robert Wood Johnson Clinical Scholars Program (JJF), the Departments of Family Medicine (JJF), Medicine (RAD), and Health Services (RAD), and the Center for Cost and Outcomes Research (RAD), University of Washington, Seattle. Address correspondence to Joshua J. Fenton, MD, RWJ Clinical Scholars Program, Box 357183, Seattle, WA 98195-7183 (e-mail: jjfenton@u.washington. edu).

This work was supported in part by grant 042251 from the Robert Wood Johnson Foundation (Investigator Award in Health Policy Research for RAD). At the time of this work, JJF was a Robert Wood Johnson Clinical Scholar. patients may ask their physicians if they should purchase one of these tests.

Many clinicians may feel uncomfortable counseling such patients, because radiologic screening tests, other than mammography, have not been recommended by physicians' organizations and are not part of usual clinical practice. Physicians may wonder: Is screening general populations with such tests likely to be effective in reducing morbidity and mortality? How should primary care physicians interpret the results if asked? How might such testing affect the health care system as a whole?

In this article, we aim to address these questions as they pertain to the population served by most family physicians. Our goal is not to present a systematic review of the literature relevant to each test. Rather, we seek to elucidate the potential clinical and ethical implications of marketing radiologic screening tests directly to consumers.

\section{Interpretation of Consumer-Purchased Screening Tests}

Interpreting a screening test requires knowledge of the pretest probability of the target disorder (the prevalence of disease in the population) and the sensitivity and specificity of the test. When the prevalence of a disease is low, as is common in primary care, the predictive value of a positive 


\begin{tabular}{|c|c|c|c|c|c|c|}
\hline Test, References & Target Condition(s) & $\begin{array}{l}\text { Prevalence } \\
(\%)^{*}\end{array}$ & $\begin{array}{l}\text { Sensitivity } \\
(\%)\end{array}$ & $\begin{array}{l}\text { Specificity } \\
(\%)\end{array}$ & PPV (\%) & Cost (US \$) \\
\hline Whole-body $\mathrm{CT}^{1,3-6}$ & Lung cancer $^{\dagger}$ & 0.5 & $55-86$ & $49-95$ & $1.2-8$ & 500 to 1300 \\
\hline $\begin{array}{l}\text { Heart scan (electron beam computed } \\
\text { tomography) }\end{array}$ & $\begin{array}{l}\text { Obstructive coronary artery } \\
\text { disease }\end{array}$ & 10 & $80^{\ddagger}$ & $40^{\ddagger}$ & 13 & 200 to 500 \\
\hline $\begin{array}{l}\text { Quantitative ultrasound of the } \\
\text { heel }^{8,13-16}\end{array}$ & Osteoporosis of the hip & $6-25$ & $71^{\ddagger}$ & $73^{\ddagger}$ & $14-47$ & 15 to 110 \\
\hline Carotid artery duplex sonography 8,17 & Carotid stenosis & $0.5-10$ & $83-86$ & 89-94 & $7-61$ & 30 to 150 \\
\hline
\end{tabular}

screening test will be low even if the test is highly sensitive and specific.

\section{Whole-Body CT}

"Whole-body" CT looks for disease throughout the chest, abdomen, and pelvis, so it has not one target condition but many. Whole-body CT is widely advertised as a screening test for cancer, so we considered its interpretation as a screening test for the most common cause of cancer mortality in the United States: lung cancer.

The prevalence of subclinical lung cancer is unknown in the general population, but $0.5 \%$ would be a conservatively high estimate. ${ }^{1}$ Randomized trials will determine whether screening CT can improve lung cancer mortality among high-risk patients, ${ }^{2}$ but nonrandomized trials suggest that CT screening may be insufficiently specific for screening low-risk populations. ${ }^{3-7}$ If a low-risk population were screened using the protocol of the Early Lung Cancer Action Project (ELCAP), the predictive value of a positive CT scan (demonstrating a noncalcified pulmonary nodule) would be only $2 \%$ (Table 1). Thus, in an unselected population, 98\% of people with a screen suspicious for lung cancer will not have cancer. The false-positive rate would be even higher if the prevalence of subclinical cancer is lower than our conservative estimate or if consumer-purchased scans have lower specificity than scans performed in the ELCAP trial.

For other cancers, the sensitivity and specificity of noncontrast CT is unknown. Yet, even if it were surprisingly accurate, noncontrast CT would be associated with a low positive predictive value because of the very low prevalence of each type of cancer in asymptomatic general populations. Although noncontrast CT can accurately diagnose abdominal aortic aneurysm and nephrolithiasis, the benefit of screening for such conditions in low-risk populations is uncertain. ${ }^{8}$

\section{Coronary Heart Scans}

Coronary heart scans generate a calcium score that is associated with coronary artery disease (Table 1). The sensitivity and specificity of heart scans in detecting obstructive coronary artery disease ( $>50 \%$ stenosis in at least one major coronary artery on angiography) depend on the calcium score that one defines as "abnormal." Higher calcium scores are more specific and less sensitive, and the opposite is true for lower calcium scores. Based on meta-analysis of 16 studies, an American College of Cardiology/American Heart Association expert panel reported that the finding of "any detectable calcium" is approximately $80 \%$ sensitive and $40 \%$ specific for obstructive coronary disease. ${ }^{9} \mathrm{Al}-$ though the prevalence of obstructive coronary disease in asymptomatic populations is unknown, we will assume for illustration a high prevalence of $10 \%$. If such a population were screened with electron-beam or helical CT of the coronary arteries, the positive predictive value of any detectable calcium would be only $13 \%$. Thus, in a low risk population, the presence of coronary calcium increases the likelihood of obstructive coronary disease by only $3 \%$.

Even if it poorly predicts obstructive coronary disease, might a positive heart scan give valuable prognostic data regarding a person's risk of developing symptomatic coronary disease? Wald et $\mathrm{al}^{10}$ 
argue that screening for a risk factor, such as coronary calcium, is worthwhile only if it powerfully predicts poor outcomes. A recent meta-analysis of data from cohort studies of asymptomatic populations screened with heart scans suggests that an abnormal heart scan is not a powerful predictor of major coronary events, such as myocardial infarction or sudden death. ${ }^{11}$ The large majority of patients with high calcium scores remain asymptomatic, and some with low calcium scores develop myocardial infarction. Current research has not proven that the calcium score adds predictive value beyond the Framingham Risk Index, which is derived from readily identified clinical data. ${ }^{12}$

\section{Heel Ultrasound}

Quantitative heel ultrasound is marketed as a screening test for osteoporosis, although it seems too inaccurate for screening in low-risk populations (Table 1). Compared with dual-energy $\mathrm{x}$-ray absorptiometry (DEXA) of skeletal sites vulnerable to fracture, heel ultrasound has a sensitivity of $62 \%$ to $81 \%$ and a specificity of $60 \%$ to $82 \%$ in diagnosing osteoporosis. ${ }^{13-16}$ Using receiver-operating characteristics, Dubois et $\mathrm{al}^{13}$ found that the sensitivity and specificity of heel ultrasound in predicting osteoporosis of the femoral neck were simultaneously maximized at $71 \%$ and $73 \%$, respectively. Consequently, in premenopausal women, for whom the prevalence of osteoporosis is $6 \%$, the positive predictive value of heel ultrasound would be only $14 \%$. Even in postmenopausal women with an osteoporosis prevalence of $25 \%$, the positive predictive value of heel ultrasound is less than $50 \%$. Furthermore, the low sensitivity of heel ultrasound means that many women with osteoporosis could be falsely reassured of normal bone density.

\section{Carotid Ultrasound}

Carotid duplex sonography screens for carotid stenosis. Although fairly sensitive and specific for important carotid stenosis, ${ }^{17}$ carotid duplex would have a positive predictive value of less than $10 \%$ in unselected persons $\leq 60$ years, assuming a $0.5 \%$ prevalence of greater than $50 \%$ stenosis. More than $90 \%$ of positive results would be false-positives. Even in octogenarians, the positive predictive value in asymptomatic persons would be unlikely to exceed $62 \%$, also leading to many false-positives. Largely because of its marginal positive predictive value, the United States Preventive Services Task
Force recommended considering carotid duplex screening only for patients with multiple cardiovascular risk factors, for whom the false-positive rate would be acceptably low. ${ }^{8}$

\section{Plausible Benefits and Harms of Screening}

Screening tests should, of course, lead to interventions or treatments that alter the course of the disease. Because screening is performed on asymptomatic people, the treatment of screen-detected disease should lead to better long-term outcomes than treatment when the disease becomes symptomatic. Moreover, the benefits of screening must outweigh its harms, which frequently stem from the evaluation of false-positive test results. Is there evidence that early detection with marketed screening tests leads to beneficial intervention?

\section{Whole-Body CT}

To date, no controlled studies have proven that CT screening of the chest, abdomen, or pelvis leads to early, effective intervention. Uncontrolled trials of chest CT screening for lung cancer in high-risk patients have shown that CT detects smaller tumors at an earlier stage than chest radiography. ${ }^{3}$ These findings give reason to hope that screening high-risk individuals with chest CT will reduce mortality from lung cancer.

Yet the results of prior lung cancer screening trials should give pause. In the Mayo Lung Project, screening chest radiography and sputum cytology also detected smaller, earlier stage tumors, and investigators initially reported increased lung cancer survival among the screened group. ${ }^{18}$ After extended follow-up, however, screening had no effect on lung cancer mortality; the seeming benefit of early detection proved to be largely attributable to a combination of lead time, length, and overdiagnosis biases. ${ }^{19}$

Likewise, screening CT of the chest, abdomen, or pelvis may advance the time of cancer diagnosis without affecting prognosis (lead-time bias) or preferentially detect indolent, less invasive disease and miss faster growing, more lethal tumors (length bias). CT screening may also uncover tumors or aneurysms that are destined to remain clinically silent. Yet, when found, these anomalies cannot be ignored and frequently prompt definitive, often invasive treatment. Although we acknowledge the plausibility of beneficial early detection with CT 
screening, controlled trials are needed to distinguish the benefit of early diagnosis from the harm of overdiagnosis and invasive treatment of "pseudodisease." 20,21

Any benefit of early detection must also outweigh the harms stemming from false-positive test results. Without question, whole-body CT screening will be associated with a considerable falsepositive rate. Many false positives will result from the chest portion alone. Assuming screening companies could replicate the test characteristics of the ELCAP trial, CT screening for lung cancer in 1000 low-risk patients would result in about 213 positive scans, with $98 \%$ false-positives. All would require follow-up high-resolution scans, and some would require multiple scans over a 2 -year span. ${ }^{22}$ When serial scans could not exclude cancer, some patients could be referred for lung biopsy, which has frequently revealed benign histology in screening trials for high-risk patients. ${ }^{4,5,23}$

False-positive tests could also result from screening with noncontrast abdominal CT. In a large series of emergency department patients receiving helical abdominal CT to evaluate for renal stones, nearly one quarter demonstrated an incidental finding for which follow-up tests seemed prudent. ${ }^{24}$ In a trial of CT screening for lung cancer, approximately $10 \%$ of patients had an indeterminate renal, adrenal, or breast finding that required further evaluation. ${ }^{23}$ Although some of these findings may occasionally lead to beneficial treatments, most have no clinical significance but require follow-up testing to prove they are harmless. Again, controlled studies are needed to assure that the harms suffered by many who would receive false-positive tests are outweighed by the benefits of far fewer whose tests would be truly positive.

A recent decision and cost-effectiveness analysis suggests that lung cancer screening with CT should await the results of clinical trials. ${ }^{25}$ In the base-case analysis, the investigators found that screening older smokers with chest CT would be quite costly but could reduce lung cancer mortality by $13 \%$. However, when the investigators assessed the potential effect of greater length and overdiagnosis biases, the harms of screening outweighed its benefits.

\section{Coronary Heart Scans}

By detecting coronary atherosclerosis early, heart scans could lead to beneficial modification of risk factors for coronary disease. However, current guidelines already advocate screening for these risk factors and aggressive intervention if present. ${ }^{26,27}$ No studies have proven an incremental benefit of heart scan screening beyond accepted practice of screening for risk factors for coronary disease.

Some patients could be reassured by a normal heart scan. Alternatively, others may be motivated by an abnormal heart scan to adopt a more salubrious lifestyle. Others with abnormal heart scans, however, might be harmed by the "label" of a new diagnosis of coronary artery disease. Negative psychological impacts of new diagnoses have been noted in other cardiovascular screening endeavors ${ }^{28}$ sometimes with worrisome social or physical ramifications. ${ }^{29,30}$ Because the prevalence of coronary calcium is high in men $>50$ years old and women $>60$ years old, ${ }^{31}$ heart scan screening will frequently diagnose subclinical coronary disease in older adults. Although evidence of the psychological effects of heart scan screening is preliminary, abnormal heart scans could have deleterious "labeling" effects in some that outweigh any plausible beneficial effects in others. ${ }^{32}$

When heart scans reveal coronary calcium, diagnostic testing for coronary artery disease will frequently ensue. ${ }^{33}$ Patients may undergo stress testing or coronary angiography, with associated risks of local vascular complications, arrhythmias, renal failure, and, in rare cases, death. ${ }^{34}$ In one cohort of asymptomatic subjects undergoing heart scan screening, $0.4 \%$ of patients had revascularization procedures based solely on the results of their heart scan. ${ }^{35}$ Thus, widespread heart scan screening could lead to coronary revascularization in many asymptomatic subjects, despite no evidence that revascularization is beneficial in patients with asymptomatic coronary disease. Finally, like other CT scans, heart scans will frequently demonstrate incidental noncardiac findings, such as pulmonary nodules or indeterminate liver lesions, which may lead to unnecessary follow-up tests or treatments. ${ }^{36}$

\section{Quantitative Ultrasound of the Heel}

The US Preventive Services Task Force recently recommended DEXA screening in women at increased risk of osteoporosis. ${ }^{37}$ The recommendation was based in part on recent randomized trials demonstrating reduced risk of clinically important fractures in women with low bone density of the femoral neck who were treated with bisphosphonates. ${ }^{38}$ Would widespread screening with heel ul- 
trasound identify a similar group of women? Not necessarily. In populations with a low prevalence of osteoporosis, many women with abnormal heel ultrasound would have normal bone mineral density of the femoral neck. Such women may be subjected to drug therapy of no benefit and the diagnostic "label" of osteoporosis. ${ }^{39}$ Alternatively, women may assume incorrectly that their bone density is normal based on a normal heel ultrasound, when in fact a hip DEXA would diagnose osteoporosis.

Screening with heel ultrasound is appealing because it is less expensive and more convenient than DEXA. In addition, cohort studies of older women have shown that abnormal heel ultrasound predicts hip fractures, even when hip DEXA does not show osteoporosis. ${ }^{40}$ Thus, it is possible that heel ultrasound could identify a subgroup of women who could benefit from antiresorptive therapy despite normal hip DEXA. This hypothesis, however, has not been tested in clinical trials. Heel ultrasound screening in general populations should await evidence that such screening reduces the risk of clinically important fracture, particularly when undertaken without appropriate pretest clinical evaluation.

\section{Carotid Artery Duplex Sonography}

The United States Preventive Services Task Force suggests that carotid duplex may have a role in screening patients at high-risk for carotid stenosis. In low-risk patients, however, the false-positive rate of carotid duplex is too high. Although the positive predictive value of carotid duplex in low-risk patients would be less than $10 \%$, patients with abnormal results would predictably seek further evaluation. Many of these would be referred for magnetic resonance angiography of the carotids, but some would conceivably be referred for carotid angiography, which poses a $1 \%$ risk of stroke. ${ }^{8}$

\section{Ethical Concerns}

\section{Retail Screening and Distributive Justice}

The bioethical principle of distributive justice holds that health care resources should be distributed as equitably as possible. Given finite resources for health services, it is unethical to provide expensive nonessential health services to one sector of society when another lacks essential services. Despite persistent inequity in the distribution of health resources in the United States, we should ask of new medical interventions, "Does this test or treatment undermine the fair distribution of health resources?"

The private purchase of a screening test could exacerbate the misdistribution of resources, because nonspecific screening tests predictably lead to further diagnostic testing, the costs of which are likely to be insured and therefore shared by the community. Most would think it is fair for someone to pay out-of-pocket for a screening test of unproven benefit. Yet, in 3 series of patients receiving screening CT of the lungs and abdomen, radiologists recommended follow-up diagnostic testing in $50 \%$ to $80 \%$ of patients. ${ }^{23,41}$ Thus, for every person who chooses to undergo CT screening, the probability seems high that their decision will result in further use of shared health resources.

If CT screening were to become widespread, the cost of follow-up diagnostic evaluation and treatment could significantly increase national health expenditures. If more funds were not allocated to cover these costs, widespread self-referral for radiologic screening tests could lead to more services for some and fewer for others, without necessarily improving the population's health as a whole.

\section{Informed Consent}

Physicians are ethically obligated to obtain informed consent from patients before tests or treatments. In the routine delivery of care, providers may obtain informed consent informally, ${ }^{42}$ but the principle still holds: the patient should understand a test or treatment's indications, benefits, risks, and limitations and make the ultimate decision regarding whether to obtain a test or treatment.

As we have reviewed, the indications, benefits, and risks of CT and ultrasound screening tests have not been elucidated by clinical research in low-risk populations. Without acknowledging plausible harms or limitations, however, many companies confidently assert the benefits of screening in advertisements. Companies can elicit credible informed consent only by acknowledging the uncertain clinical benefits and possible harms of radiologic screening tests.

\section{Responding to the Trend}

Rapid growth in the screening test industry requires a reasoned response from physicians and policy-makers. National and regional medical asso- 
ciations should discourage the marketing of screening tests of unproven benefit and alert patients to the potential hazards of indiscriminate testing among low-risk populations. A statement opposing whole-body CT screening from the American College of Radiology received substantial media coverage. ${ }^{43}$ Similar statements from other physicians' groups could engender healthy skepticism among consumers and rebut industry advertisements, which emphasize only the positive outcomes of testing.

Individual physicians should warn about the risks of indiscriminate screening tests among lowrisk populations. Some patients may be dissuaded from testing if their physicians advise against it. Before physicians will advise against a test, however, they must understand its potential harms. Many physicians may assume that advanced imaging tests are accurate, informative, and better than no screening at all. Professional societies may therefore have a crucial role in disseminating information about the limitations of marketed tests. The American Academy of Family Physicians and the American College of Physicians-American Society of Internal Medicine both issue recommendations regarding preventive health services. As the professional academies representing most primary care physicians serving adults, both should alert members about the potential hazards of radiologic screening without appropriate pretest clinical evaluation.

Although regulatory oversight could protect consumers from the hazards of indiscriminate testing, no federal agency currently has a mandate to regulate use of radiologic machinery once the United States Food and Drug Administration (FDA) has certified it for marketing and distribution. Any expansion of the FDA's mandate would require new federal legislation, which currently seems improbable. Some states require a physicians' prescription before radiologic testing. ${ }^{44}$ Other states may consider legislation requiring physician referral for radiologic testing if growth in the screening industry results in substantially increased health care costs. At the federal level, the Federal Trade Commission should closely monitor company advertisements and discourage misrepresentation of the benefits of screening.

One might suggest that health insurers refuse to pay for tests and treatments stemming from an enrollee's purchase of a radiologic screening test.
Such a policy, however, could be difficult to enforce, because once abnormalities are uncovered at screening, clinicians may find them impossible to ignore. When confronted by a patient with an abnormal screening test, most physicians will feel obligated to fully evaluate the abnormality. If a physician orders further tests, the insurance company may be unable to prove that the physician ordered follow-up tests solely based on the screening test, rather than other clinical indications. Such a policy could also pose legal risks. If a single patient believes that administrative barriers erected by the insurer forestalled timely diagnosis and treatment, he or she could sue the insurer for damages that far exceed the costs of follow-up tests for many abnormal screening exams.

In summary, current evidence does not support widespread screening with most of the radiologic tests now marketed to consumers. Although advertisements dramatize the benefits of testing, these tests have the potential to cause harm, especially when falsely positive. Patients should generally avoid radiologic screening tests until the tests have been appropriately evaluated in controlled studies and recommended by unbiased national panels. $\mathrm{Al}-$ though regulatory measures could protect consumers from the hazards of screening, federal or state legislation restricting the marketing of radiologic screening tests is unlikely to be passed in the near term. Physicians and their professional organizations, therefore, have a crucial role in publicizing the uncertain benefits and potential hazards of radiologic screening tests.

We thank Jerry Jarvik, MD, MPH and Benjamin Littenberg, $\mathrm{MD}$, for helpful comments on earlier versions of this article.

\section{References}

1. Wingo PA, Tong T, Bolden S. Cancer statistics, 1995. CA Cancer J Clin 1995;45:8-3.

2. Gohagan JK, Prorok PC, Hayes RB, Kramer BS. The Prostate, Lung, Colorectal and Ovarian (PLCO) cancer screening trial of the National Cancer Institute: history, organization, and status. Control Clin Trials 2000;21 Suppl 6:251S-72S.

3. Henschke CI, McCauley DI, Yankelevitz DF, et al. Early Lung Cancer Action Project: overall design and findings from baseline screening. Lancet 1999; 354:99-105.

4. Sone S, Li F, Yang ZG, et al. Results of three-year mass screening programme for lung cancer using 
mobile low-dose spiral computed tomography scanner. Br J Cancer 2001;84:25-32.

5. Sobue T, Moriyama N, Kaneko M, et al. Screening for lung cancer with low-dose helical computed tomography: anti-lung cancer association project. J Clin Oncol 2002;20:911-20.

6. Swensen SJ, Jett JR, Sloan JA, et al. Screening for lung cancer with low-dose spiral computed tomography. Am J Respir Crit Care Med 2002;165:508-13.

7. Patz EF Jr, Goodman PC, Bepler G. Screening for lung cancer. N Engl J Med 2000;343:1627-33.

8. United States Preventive Services Task Force. Guide to clinical preventive services. Alexandria (VA): International Medical Publishing; 1996.

9. O'Rourke RA, Brundage BH, Froelicher VF, et al. American College of Cardiology/American Heart Association Expert Consensus document on electron-beam computed tomography for the diagnosis and prognosis of coronary artery disease. Circulation 2000;102:126-40.

10. Wald NJ, Hackshaw AK, Frost CD. When can a risk factor be used as a worthwhile screening test? BMJ 1999;319:1562-5.

11. O'Malley PG, Taylor AJ, Jackson JL, Doherty TM, Detrano RC. Prognostic value of coronary electronbeam computed tomography for coronary heart disease events in asymptomatic populations. Am J Cardiol 2000;85:945-8.

12. Detrano RC, Wong ND, Doherty TM, et al. Coronary calcium does not accurately predict near-term future coronary events in high-risk adults. Circulation 1999;99:2633-8.

13. Dubois EFL, van den Bergh JPW, Smals AGH, van de Meerendonk CWD, Zwinderman AH, Schweitzer DH. Comparison of quantitative ultrasound parameters with dual energy X-ray absorptiometry in pre- and postmenopausal women. Neth J Med 2001;58:62-70.

14. Ayers M, Prince M, Ahmadi S, Baran DT. Reconciling quantitative ultrasound of the calcaneus with $\mathrm{X}$-ray-based measurements of the central skeleton. J Bone Miner Res 2000;15:1850-5.

15. Fordham JN, Chinn DJ, Kumar N. Identification of women with reduced bone density at the lumbar spine and femoral neck using BMD at the os calcis. Osteoporos Int 2000;11:797-802.

16. Rosenthall L, Tenenhouse A, Caminis J. A correlative study of ultrasound calcaneal and dual-energy X-ray absorptiometry bone measurements of the lumbar spine and femur in 1000 women. Eur J Nucl Med 1995;22:402-6.

17. Blakeley DD, Oddone EZ, Hasselblad V, Simel DL, Matchar DB. Noninvasive carotid artery testing: a meta-analytic review. Ann Intern Med 1995;122: 360-7.

18. Sanderson DR. Screening: lung cancer. Chest 1986; 89(4 suppl):324S-26S.

19. Marcus PM, Bergstralh EJ, Fagerstrom RM, et al.
Lung cancer mortality in the Mayo Lung Project: impact of extended follow-up. J Natl Cancer Inst 2000;92:1308-16.

20. Black WC, Welch HG. Advances in diagnostic imaging and overestimations of disease prevalence and the benefits of therapy. N Engl J Med 1993;328: 1237-43.

21. Black WC. Overdiagnosis: an underrecognized cause of confusion and harm in cancer screening. J Nat Cancer Inst 2000;92:1280-2.

22. Aberle DR, Gamsu G, Henschke CI, Naidich DP, Swensen SJ. A consensus statement of the Society of Thoracic Radiology: screening for lung cancer with helical computed tomography. J Thorac Imaging 2001;16:65-8.

23. Swensen SJ. CT screening for lung cancer. AJR Am J Roentgenol 2002;179:833-6.

24. Messersmith WA, Brown DF, Barry MJ. The prevalence and implications of incidental findings on ED abdominal CT scans. Am J Emerg Med 2001;19: 479-81.

25. Mahadevia PJ, Fleisher LA, Frick KD, Eng J, Goodman SN, Powe NR. Lung cancer screening with helical computed tomography in older adult smokers: a decision and cost-effectiveness analysis. JAMA 2003;289:313-22.

26. Joint National Committee on Prevention, Detection, Evaluation, and Treatment of High Blood Pressure. The Sixth Report of the Joint National Committee on prevention, detection, evaluation, and treatment of high blood pressure (JNC VI). NIH publication 98-4080. Bethesda (MD): National Institutes of Health, National Heart, Lung, and Blood Institute; 1997.

27. National Cholesterol Education Program. Detection, evaluation, and treatment of high blood cholesterol in adults (Adult Treatment Panel III). NIH publication no. 01-3670. Bethesda (MD): National Institutes of Health, National Heart, Lung, and Blood Institute; 2001. Available at: URL: http://www.nhlbi.nih.gov/guidelines/cholesterol/ atp3_rpt.htm

28. Bergman AB, Stamm SJ. The morbidity of cardiac nondisease in schoolchildren. N Engl J Med 1967; 276:1008-13.

29. Haynes RB, Sackett DL, Taylor DW, Gibson ES, Johnson AL. Increased absenteeism from work after detection and labeling of hypertensive patients. N Engl J Med 1978;299:741-4.

30. Taylor DW, Haynes RB, Sackett DL, Gibson ES. Long-term follow-up of absenteeism among working men following the detection and treatment of their hypertension. Clin Invest Med 1981;4:173-7.

31. Hoff JA, Chomka EV, Krainik AJ, Daviglus M, Rich S, Kondos GT. Age and gender distributions of coronary artery calcium detected by electron beam tomography in 35,246 adults. Am J Cardiol 2001;87: 1335-9. 
32. Wong ND, Detrano RC, Diamond G, et al. Does coronary artery screening by electron beam computed tomography motivate potentially beneficial lifestyle behaviors? Am J Cardiol 1996;78:1220-3.

33. Taylor AJ, O'Malley PG. Self-referral of patients for electron-beam computed tomography to screen for coronary artery disease. N Engl J Med 1998;339: 2018-20.

34. Johnson LW, Lozner EC, Johnson S, et al. Coronary arteriography 1984-1987: a report of the Registry of the Society for Cardiac Angiography and Interventions. I. Results and complications. Cathet Cardiovasc Diagn 1989;17:5-10.

35. Arad Y, Spadaro LA, Goodman K, Newstein D, Guerci AD. Prediction of coronary events with electron beam computed tomography. J Am Coll Cardiol 2000;36:1253-60.

36. Horton KM, Post WS, Blumenthal RS, Fishman EK. Prevalence of significant noncardiac findings on electron-beam computed tomography coronary calcium screening examinations. Circulation 2002;106: 532-4.

37. Nelson HD, Helfand M, Woolf SH, Allan JD. Screening for postmenopausal osteoporosis: a review of the evidence for the U.S. Preventive Services Task Force. Ann Intern Med 2002;137:529-41.

38. Cummings SR, Black DM, Thompson DE, et al.
Effect of alendronate on risk of fracture in women with low bone density but without vertebral fractures: results from the Fracture Intervention Trial. JAMA 1998;280:2077-82.

39. Rubin SM, Cummings SR. Results of bone densitometry affect women's decisions about taking measures to prevent fractures. Ann Intern Med 1992; 116:990-5.

40. Bauer DC, Gluer CC, Cauley JA, et al. Broadband ultrasound attenuation predicts fractures strongly and independently of densitometry in older women. A prospective study. Study of Osteoporotic Fractures Research Group. Arch Intern Med 1997;157: 629-34.

41. Peck P. Full-body CT screening divides radiology community. Available at: URL: http://www.medscape.com/viewarticle/445636.

42. Braddock CH, Edwards K, Hasenburg NM, Laidley TL, Levinson W. Informed decision making in outpatient practice: time to get back to basics. JAMA 1999;282:2313-20.

43. ACR statement on CT screening exams. Reston (VA): American College of Radiology; 2000. Available at: URL: http://www.acr.org/departments/ pub_rel/press_releases/total-bodyCT.html.

44. Holtz A. Regulatory ins and outs of self-referral for CT scans. Oncology Times 2003;25:8-11. 